\title{
Knockdown of WAVE1 enhances apoptosis of leukemia cells by downregulating autophagy
}

\author{
ZHAOXIA ZHANG $^{1 *}$, BENQING WU $^{1 *}$, WENWEN CHAI $^{2}$, LIZHI CAO $^{3}$, \\ YANGPING WANG ${ }^{3}$, YAN YU ${ }^{3}$ and LIANGCHUN YANG ${ }^{3}$ \\ ${ }^{1}$ Department of Pediatrics, Shenzhen People's Hospital, Shenzhen, Guangdong 518020; \\ ${ }^{2}$ Department of Nuclear Medicine, Hu Nan Cancer Hospital and Affiliated Cancer Hospital of Xiangya School of Medicine, \\ Central South University, Changsha, Hunan $410008 ;{ }^{3}$ Department of Pediatrics, Xiangya Hospital, \\ Central South University, Changsha, Hunan 410008, P.R. China
}

Received January 16, 2016; Accepted February 20, 2016

DOI: 10.3892/ijo.2016.3446

\begin{abstract}
Chemoresistance of leukemia constitutes a great challenge for successful treatment of leukemia. Autophagy has recently attracted increasing attention for its role in conferring resistance to various conventional anti-neoplastic regiments. In the present study, the authors showed that WAVE1, a member of WASP family verprolin-homologous proteins, is a critical regulator of chemoresistance during autophagy. It is positively correlated with clinical status in pediatric acute myeloblastic leukemia (AML) and leukemia cell lines. The knockdown of WAVE1 expression decreased autophagy was accompanied by an upregulation of autophagic marker microtubule-associated protein light chain 3 (LC3)-II, a degradation of SQSTM1/sequestosome 1 (p62) and the formation of autophagosomes. Moreover, a suppression of WAVE1 expression increased the sensitivity of leukemia cells to chemotherapy and apoptosis, and depletion of WAVE1 expression promoted the translocation of $\mathrm{Bcl}-2$ from mitochondria into the cytoplasm. In addition, a knockdown of PI3K-III expression significantly inhibited WAVE1-mediated autophagy. Furthermore, suppression of WAVE1 expression blocked the interactions between Beclin1 and PI3K-III and the disassociation of Beclin1-Bcl-2 during enhanced autophagy. The above results suggested that WAVE1 is a critical pro-autophagic protein capable of enhancing cell survival and regulating chemoresistance in leukemia cells potentially through the Beclin1/Bcl-2 and Beclin1/PI3K-III complex-dependent pathways.
\end{abstract}

Correspondence to: Dr Liangchun Yang, Department of Pediatrics, Xiangya Hospital, Central South University, Changsha, Hunan 410008, P.R. China

E-mail: yangliangchung@163.com

*Contributed equally

Key words: WAVE1, autophagy, leukemia, chemoresistance

\section{Introduction}

As a malignant disease of bone marrow and blood, leukemia is the most common form of cancer in children. Pediatric acute myeloid leukemia (AML) represents $15-20 \%$ of all pediatric acute leukemias. The clinical outcomes of pediatric AML have improved significantly over the past few decades so that the long-term survival rate stands at $\sim 70 \%$. Improvement is due to intensification of chemotherapeutic regimens, optimal riskgroup stratification, timely salvage for relapse and enhanced supportive cares (1-3). Despite intensive treatment, $\sim 30 \%$ of pediatric patients relapsed with poor outcomes. In addition, $30-40 \%$ of patients survived in the largest and most recent series $(4,5)$. Chemoresistance of leukemia cells constitutes a great challenge for successful therapeutics. Autophagy has been shown to played important roles in conferring resistance to chemotherapy, radiation therapy and immunotherapy in cancer cells $(6,7)$. However, exact molecular mechanisms by which autophagy induces drug resistance in cancer cells, specifically leukemia cells, have been poorly defined.

The Wiskott-Aldrich syndrome (WAS) protein (WASP) and WASP family verprolin-homologous protein (WAVE) family is composed of 5 members, i.e. WASP, N-WASP, WAVE1, WAVE2 and WAVE3. They serve as key links between GTPases and actin cytoskeleton (8). Essential for cell morphologic changes, motility and apoptosis, cytoskeleton reorganization is also an important regulator of autophagy (9-11). Actin microfilaments and microtubules are vital for initial formation of autophagosomes in starved cells (11). Moreover, cytoskeleton regulatory proteins such as dynein, myosin and kinesin play important regulatory roles during autophagy (12-16). Similar to other intracellular trafficking events, autophagosomal movement employs microtubule-dependent machinery in mammalian cells $(17,18)$. Moreover, these observations have provided evidence for the relevance of cytoskeleton during autophagy.

WAVE1 (also known as Scar1), a suppressor of cyclic AMP receptor 1, was first identified as a regulator of actin cytoskeleton through interactions with Arp2/3 downstream of Rac $(19,20)$. WAVE1 is expressed most abundantly in murine brain tissue and leukemia cells and at extremely low 
levels in other tissues including heart, liver, lung, kidney, pancreas and peripheral blood $(10,21)$. In addition to its roles in the nervous system development and mammalian fertilization, WAVE1 also functions as an important molecule for regulating tumor development, tumor invasion and metastasis (22-24). Our previous studies have shown that WAVE1 was involved in multidrug resistance and oxidative stress of human leukemia cells and functioned as a negative regulator of apoptosis $(10,25,26)$. As autophagy and apoptosis are currently regarded as different aspects of the same cell death continuum, their regulations become intimately connected. In addition, the same regulators could control both apoptosis and autophagy (27). In light of the fact that cytoskeleton reorganization is also an important regulator of autophagy, the role of WAVE1 in leukemia cell apoptosis is expected to shed some light on its role in autophagy.

It was shown in the present study that WAVE1 was overexpressed in both human hematological cancer cell lines and primary BMMCs from patients with pediatric leukemia. WAVE1 expression was positively correlated with clinical status in pediatric leukemia. Suppression of WAVE1 expression blocked drug-induced autophagic reactions and increased the sensitivity of leukemia cells to anti-neoplastic agents. Furthermore, our data suggested a regulatory role for WAVE1 during autophagy through a formation of Beclin1-PI3K-III complex and a disassociation of Bcl-2-Beclin1 complex. Thus, WAVE1 is a potential drug target of therapeutic interventions for leukemia.

\section{Materials and methods}

Reagents and antibodies. The antibodies to WAVE1 and p62 were purchased from Santa Cruz Biotechnology (Santa Cruz, CA, USA); antibodies to LC3, actin and tubulin from Sigma Inc. (St. Louis, MO, USA); antibody to mitochondria HSP70 (mHSP70) from Abcam (Cambridge, MA, USA); antibodies to class III phosphoinositide 3-kinase (PI3K-III), p-4EBP1, Beclin1 and Bcl-2 from Cell Signaling Technology (Danvers, MA, USA); 3-methyladenine (3-MA), vincristine (VCR), cytosine arabinoside (Ara-C), adriamycin (ADM), E64D and pepstatin from Sigma.

Cell culture. HL-60 acute promyelocytic leukemia cells, K562 chronic myelogenous leukemia cells, THP-1 acute monocytic leukemia cells, adriamycin-resistant HL-60/ADR cells, A549 human lung cancer cells, human umbilical vein endothelial cells, human lung A549 cancer cells and CNE2 nasopharyngeal carcinoma cells (Xiangya School of Medicine Type Culture Collection, Xiangya, China) were cultured in RPMI-1640 or DMEM medium with $10 \%$ heat-inactivated fetal bovine serum (FBS) in $5 \% \mathrm{CO}_{2}$ and $95 \%$ ambient air.

Gene transfection and RNAi. WAVE1 small hairpin RNA (shRNA) lentiviral knockdown (GeneCopoeia, Guangzhou, China) or shRNA non-target control (NTC) were packaged with HIV-based packaging mix (GeneCopoeia) for infecting HL-60 cells to establish cells constitutively repressing WAVE1. Stable clones were selected by puromycin. PI3K-III shRNA from Sigma were constructed with FuGENE HD transfection reagent (Roche Applied Science, Stockholm, Sweden) according to the manufacturer's instructions.
Cell viability assay. After drug dosing, cell viability was evaluated by Cell Counting kit-8 (CCK-8) (Dojindo Molecular Technologies, Tokyo, Japan) according to the manufacturer's instructions.

Reverse transcription-polymerase chain reaction (RT-PCR). Total RNA was isolated by TRIzol reagent (Invitrogen, Carlsbad, CA, USA) according to the manufacturer's instructions. RNA concentration and purity were measured with a spectrophotometer at A260 and A260/280, respectively. RNA was reverse-transcribed into cDNA using a Primescript ${ }^{\mathrm{TM}}$ RT reagent kit (Invitrogen) according to the manufacturer's instructions. The sequences of primers used were as follows: for $\beta$-actin: forward, 5'-TCCTTCCTGGGCATGGAGTC-3' and reverse, 5'-GTAACGCAACTAAGTCATAGTC-3'. For WAVE1: forward, 5'-TCTGGGCTACATCCAACTCC-3' and reverse, 5'-CCTGTTCACGCTGCTCTTCT-3'. $\beta$-actin was used as an internal control for evaluating the relative expressions of WAVE1. The conditions of polymerase chain reaction (PCR) for WAVE1 were as follows: denaturation at $94^{\circ} \mathrm{C}$ for $2 \mathrm{~min}$, followed by 30 cycles of $94^{\circ} \mathrm{C}$ for $30 \mathrm{sec}, 56^{\circ} \mathrm{C}$ for $30 \mathrm{sec}$ ( $\beta$-actin, $50^{\circ} \mathrm{C}$ for $30 \mathrm{sec}$ ), $72^{\circ} \mathrm{C}$ for $30 \mathrm{sec}$ and ultimately by a 5-min elongation at $72^{\circ} \mathrm{C}$. The PCR products were analyzed with $1.0 \%$ agarose gel electrophoresis, ethidium bromidestained, photographed and scanned by Band Leader software for grey scale semi-quantitative analysis.

Western blot analysis. After rinsing with phosphate-buffer solution (PBS), the cells were collected, resuspended in lysis buffer (Beyotime Institute of Biotechnology, Beijing, China) and maintained on ice for $15 \mathrm{~min}$. Cell extracts were cleared by microcentrifugation at $14,000 \mathrm{xg}$ for $30 \mathrm{~min}$ at $4^{\circ} \mathrm{C}$. Whole cell lysate was separated by $8 \%(10 \% / 12 \%)$ sodium dodecyl sulfate-polyacrylamide gel electrophoresis (SDS-PAGE) and electrophoretically transferred onto polyvinylidene difluoride (PVDF) blotting membrane (Beyotime Institute of Biotechnology). After blocking with 5\% non-fat dry milk in TBST (50 mM Tris pH 7.5, $100 \mathrm{mM} \mathrm{NaCl,} \mathrm{0.15 \%} \mathrm{Tween-20),}$ the membranes were incubated with diluted primary antibodies for $12 \mathrm{~h}$ at $4^{\circ} \mathrm{C}$ and washed thrice with TBST for $10 \mathrm{~min}$. After incubating for $12 \mathrm{~h}$ at $4^{\circ} \mathrm{C}$ with various secondary antibodies, detection was made with enhanced chemiluminescence (ECL) reagents (Pierce, Rockford, IL, USA) after rinsing thrice with TBST for $10 \mathrm{~min}$. The membranes were exposed to X-ray film and the expressions of targeted proteins quantified by detecting specific bands. In addition, BandScan 5.0 system was used for quantifying and analyzing each specific blotting band (16).

Immunoprecipitation. Cells were lysed at $4^{\circ} \mathrm{C}$ in ice-cold lysis buffer (50 mM Tris-HCl, pH 7.4, containing $150 \mathrm{mM} \mathrm{NaCl}$, $1 \%$ NP-40, $0.5 \%$ nadeoxycholate, $0.1 \%$ SDS, protease inhibitor cocktail) and cell lysates centrifugated at $12000 \mathrm{x}$ g for $10 \mathrm{~min}$. The concentrations of proteins in supernatant were determined by bicinchoninic acid (BCA) assay. Prior to immunoprecipitation, the samples containing equal amounts of proteins were pre-cleared with protein A or protein $\mathrm{G}$ agarose/sepharose (Santa Cruz Biotechnology, Santa Cruz, CA, USA) $\left(4^{\circ} \mathrm{C}, 3 \mathrm{~h}\right)$ and subsequently incubated with various irrelevant $\mathrm{IgG}$ or specific antibodies $(5 \mathrm{mg} / \mathrm{ml})$ in the presence of protein A or $\mathrm{G}$ agarose/sepharose beads for $2 \mathrm{~h}$ or overnight at $4^{\circ} \mathrm{C}$ with 

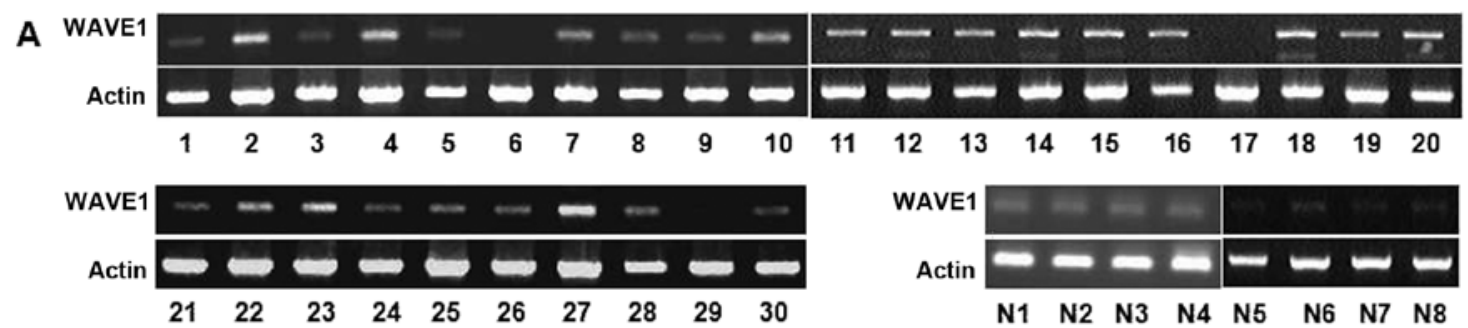

B
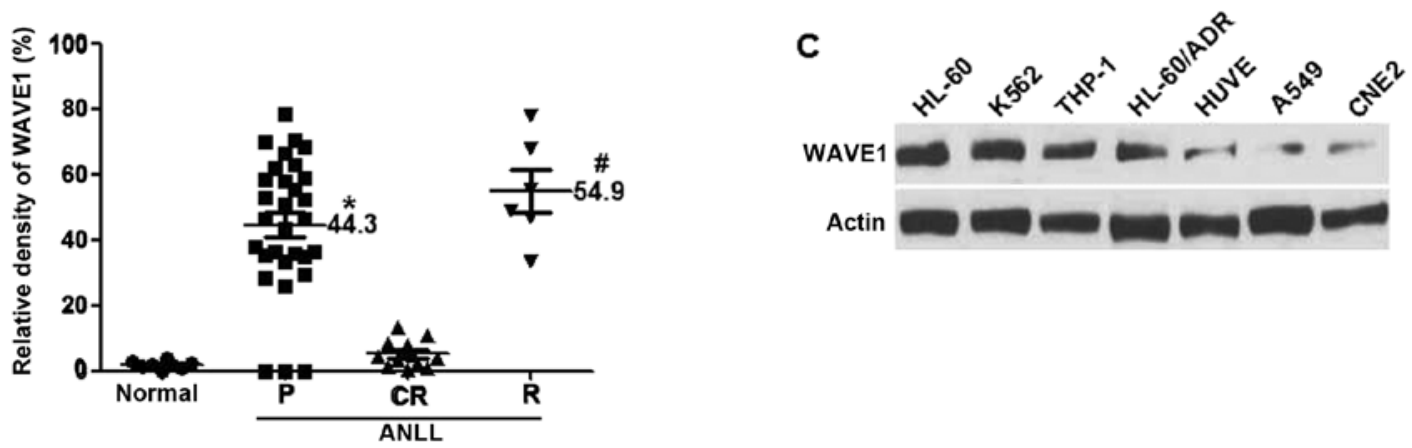

Figure 1. Expression of WAVE1 in pediatric AML patients and leukemia cell lines. (A) Expression of WAVE1 at mRNA level in different samples. Total mRNA was extracted from BMMCs of 30 patients and 8 normal healthy subjects. WAVE1 level was determined by RT-PCR. Each patient number corresponds to that shown in Table I. Actin was used as a loading control. (B) Relative expression levels of WAVE1 in childhood AML patients. Total mRNA was extracted from BMMCs of normal healthy subjects or patients, and WAVE1 level was determined by the relative optical intensity of bands by RT-PCR. Each dot represents relative WAVE1 level in each individual sample. ${ }^{*} \mathrm{P}<0.05$ vs. $\mathrm{N} ;{ }^{*} \mathrm{P}<0.05$ vs. CR. N, normal healthy subject; $\mathrm{P}$, primary; CR, complete remission; R, relapse. (C) WAVE1 was overexpressed in blood cancer cell lines. Western blot analysis of WAVE1 in various human cancer cell lines. Actin was used as a loading control.

gentle vortexing. After incubation, agarose/sepharose beads were rinsed thoroughly with PBS, and the proteins were eluted by boiling in $2 \mathrm{X}$ SDS sample buffer prior to SDS-PAGE.

Apoptosis assays. Cellular apoptosis was assessed by FITC Annexin V apoptosis detection kit [Annexin V-FITC, propidium iodide (PI) solution and Annexin $\mathrm{V}$ binding buffer]. This assay involved staining cells with Annexin V-FITC (a phospholipid-binding protein binding to disrupted cell membranes) in combination with PI (a vital dye binding to DNA penetrating into apoptotic cells). Flow cytometry (FACS) was performed for determining the percentage of apoptotic cells (Annexin $\left.\mathrm{V}^{+} / \mathrm{PI}\right)$.

Electron microscopy. After collection, NB4 cells were fixed in $2.5 \%$ glutaraldelhyde for at least $3 \mathrm{~h}$ and subsequently treated with $2 \%$ paraformaldehyde at room temperature for $60 \mathrm{~min}$, $0.1 \%$ glutaraldehyde in $0.1 \mathrm{M}$ sodium cacodylate for $2 \mathrm{~h}$ and post-fixation with $1 \%$ osmium tetroxide $\left(\mathrm{OsO}_{4}\right)$ for $1.5 \mathrm{~h}$. After the second rinsing, the samples were dehydrated with graded acetone and ultimately embedded in Quetol 812. Ultrathin sections were observed under Hitachi H7500 electron microscope (Hitachi, Tokyo, Japan).

Caspase activity assay. Caspase-3 activity was assayed by caspase-3 colorimetric assay kit (Calbiochem, Berlin, Germany) according to the manufacturer's instructions.

Statistical analysis. The quantitative data were presented as means \pm standard deviation. In addition, analysis was performed with specified statistical methods using GraphPad Prism (version 5.04). For calculating P-value, two-tailed parameters with a confidence interval of $95 \%$ were used. A P-value $<0.05$ was considered to indicate a statistically significant result.

\section{Results}

Correlation between WAVE1 expression and clinical status in pediatric AML. A total of 30 patients aged 1-13 years with newly diagnosed AML at our department were enrolled from January 2008 to December 2014. There were 17 males and 13 females (Table I). The median age was 6 years (range, 1-13 years). According to the French-American-British (FAB) classification scheme, the types were M0 $(n=1)$, M1 $(n=1)$, M2 $(n=16)$, M4 $(n=7)$, M5 $(n=4)$ and M7 $(n=1)$. At the time of diagnosis, the median count of white blood cells (WBC) was $14.9 \times 10^{9} / 1$ (range, $0.8-102.7 \times 10^{9} / 1$ ), median hemoglobin level $66.5 \mathrm{~g} / 1$ (range, 31-241 g/l) and median platelet count $31.2 \times 10^{9} / 1$ (range, $2-104.6 \times 10^{9} / 1$ ). The median relative level of WAVE1 mRNA expression was 0.4635 (range, 0.0-0.781), and $22(73.3 \%)$ achieved a complete remission (CR) with 1 or 2 induction chemotherapies. The survival outcomes revealed that $16 / 30$ patients $(48.4 \%)$ achieved CR after 6-month chemotherapy, and 10 of them (33.3\%) died. Relapsing and refractory leukemias were the commonest cause of mortality $(n=8)$, followed by infection $(n=1)$ and hemorrhage $(n=1)$. All of them received anthracycline and cytarabine-based chemotherapeutic regimens, and 4 patients received hematopoietic stem cell transplantation (HSCT) after induction therapy.

WAVE1 expression patterns were detected by RT-PCR in 30 newly-diagnosed AML patients and 8 normal healthy subjects (Fig. 1A). The relative WAVE1 mRNA levels were summarized in Table I. There was a general trend for a higher 
Table I. Clinical characteristics of 30 pediatric AML patients.

\begin{tabular}{|c|c|c|c|c|c|c|c|}
\hline Subtype & No. & $\begin{array}{c}\text { Age } \\
\text { (years) }\end{array}$ & Gender & $\begin{array}{l}\text { Initial leukocyte } \\
\text { count }\left(\times 10^{9} / 1\right)\end{array}$ & $\begin{array}{c}\text { BM evaluation after } 1 / 2 \\
\text { induction chemotherapies }\end{array}$ & $\begin{array}{l}\text { Relative WAVE1 } \\
\text { mRNA expression }\end{array}$ & $\begin{array}{l}\text { Survival } \\
\text { outcome }\end{array}$ \\
\hline M2 & 1 & 6 & Male & 6.5 & $\mathrm{CR}$ & 0.335 & CR \\
\hline M2 & 2 & 8 & Female & 63.2 & $\mathrm{CR}$ & 0.589 & HSCT \\
\hline M4 & 3 & 2 & Male & 7.8 & IR (BM blasts, 22.8\%) & 0.321 & Deceased (hemorrhage) \\
\hline M2 & 4 & 5 & Female & 24.7 & $\mathrm{CR}$ & 0.582 & Deceased (infection) \\
\hline M1 & 5 & 1 & Male & 3.6 & $\mathrm{CR}$ & 0.378 & $\mathrm{CR}$ \\
\hline M2 & 6 & 3 & Male & 1.4 & CR & 0 & CR \\
\hline M2 & 7 & 11 & Male & 18.5 & IR (BM blasts, $10.4 \%$ ) & 0.434 & CR \\
\hline M5 & 8 & 6 & Male & 26.3 & IR (BM blasts, $8.3 \%$ ) & 0.391 & $\mathrm{CR}$ \\
\hline M2 & 9 & 10 & Female & 2.1 & $\mathrm{CR}$ & 0.361 & CR \\
\hline M4 & 10 & 13 & Female & 46.7 & CR & 0.528 & Deceased (relapsing) \\
\hline M4 & 11 & 8 & Male & 34.6 & CR & 0.551 & HSCT \\
\hline M4 & 12 & 3 & Male & 10.2 & CR & 0.580 & HSCT \\
\hline M5 & 13 & 5 & Female & 19.8 & CR & 0.628 & Deceased (refractory) \\
\hline M2 & 14 & 7 & Female & 102.7 & IR (BM blasts, $11 \%$ ) & 0.684 & Deceased (relapsing) \\
\hline M2 & 15 & 6 & Female & 73.1 & $\mathrm{CR}$ & 0.617 & Deceased (refractory) \\
\hline M2 & 16 & 13 & Male & 90.5 & IR (BM blasts, 37\%) & 0.699 & Deceased (refractory) \\
\hline M2 & 17 & 9 & Male & 4.7 & CR & 0 & CR \\
\hline M5 & 18 & 3 & Male & 88.4 & CR & 0.781 & Deceased (relapsing) \\
\hline M5 & 19 & 5 & Male & 41.3 & CR & 0.508 & CR \\
\hline M4 & 20 & 2 & Female & 9.7 & CR & 0.702 & Deceased (refractory) \\
\hline M2 & 21 & 7 & Male & 0.8 & $\mathrm{CR}$ & 0.257 & CR \\
\hline M2 & 22 & 4 & Male & 47.6 & IR (BM blasts, $15 \%$ ) & 0.463 & $\mathrm{CR}$ \\
\hline M0 & 23 & 7 & Female & 55.8 & $\mathrm{CR}$ & 0.523 & HSCT \\
\hline M2 & 24 & 11 & Female & 3.7 & CR & 0.295 & $\mathrm{CR}$ \\
\hline M2 & 25 & 5 & Female & 2.5 & CR & 0.357 & $\mathrm{CR}$ \\
\hline M4 & 26 & 2 & Female & 11.3 & CR & 0.346 & CR \\
\hline M7 & 27 & 12 & Male & 57.6 & IR (BM blasts, $75 \%$ ) & 0.663 & Deceased (relapsing) \\
\hline M2 & 28 & 10 & Male & 2.5 & CR & 0.362 & $\mathrm{CR}$ \\
\hline M2 & 29 & 4 & Female & 1.0 & IR (BM blasts, 26\%) & 0 & CR \\
\hline M4 & 30 & 8 & Male & 3.9 & CR & 0.284 & CR \\
\hline
\end{tabular}

FAB classification of AML: M0, M1, M2, M3, M4, M5, M6 and M7; BM, bone marrow; CR, complete remission (BM blast $\geq 5 \%$ ); IR, incomplete remission (BM blast $\geq 5 \%$ ); HSCT, hematopoietic stem cell transplantation. The relative optical intensity of bands between WAVE1 and $\beta$-actin (WAVE1/ $\beta$-actin).

incidence of relapsing or refractory leukemia in WAVE1 high-expression patients. Also a poor prognosis was found in patients with leucocytosis at diagnosis (Table II). Moreover, the relative WAVE1 mRNA expression levels were examined for 30 pediatric AML patients at varying clinical status. Higher levels of WAVE1 expression were found in bone marrow mononuclear cells (BMMCs) from patients with primary $(n=30)$ and relapsing $(n=6)$ leukemias (Fig. 1B). By contrast, WAVE1 was not detectable in BMMCs from CR patients $(n=12)$ or normal healthy subjects $(n=8)$ (Fig. 1B). Thus WAVE1 was well-correlated with the clinical status of pediatric AML.

Furthermore, the expression levels of WAVE1 were determined by western blot analysis in 4 leukemia cell lines of
HL-60, K562, HL-60/ADR and THP-1, their levels were all upregulated (Fig. 1C). In contrast, the constitutive expression levels of WAVE1 were noticeably lower in non-hematological cancer cell lines, including human lung A549 cancer cells, human umbilical vein endothelial cells and CNE2 nasopharyngeal carcinoma cells. Thus, a different role of WAVE1 is implicated for leukemic tumorigenesis.

Targeting shRNA-mediated silencing of WAVE1 expression. A specific shRNA against WAVE1 was transferred into HL-60 for knocking down the expression of WAVE1. The cellular transfection efficiency was determined by visualizing eGFP-positive cells under microscope and confirmed by flow cytometry (Fig. 2A and B). Moreover, RT-PCR and western 
Table II. Results of various patient variables during BM evaluation.

\begin{tabular}{lccc}
\hline & CR group $(\mathrm{n}=16)$ & Relapsing/refractory group $(\mathrm{n}=8)^{\text {P-value }}$ \\
\hline WAVE1 & $0.298(1-0.508)^{\mathrm{b}}$ & $0.662(0.530-0.780)$ & 0.00 \\
WBCs $\left(\mathrm{x} 10^{9} / \mathrm{l}\right)$ at diagnosis & $11.1(0.8-47.6)$ & $61.1(9.7-102.7)$ & 0.00 \\
$\mathrm{Hb}(\mathrm{g} / \mathrm{l})$ at diagnosis & $70.1(31-105)$ & $84(58-169)$ & 0.276 \\
Platelet $\left(\mathrm{x} 10^{9} / \mathrm{l}\right)$ at diagnosis & $48.5(2-149)$ & $49.3(5-106)$ & 0.793 \\
Age at diagnosis (years) & $6.3(1-11)$ & $7.6(2-13)$ & 0.438 \\
\hline
\end{tabular}

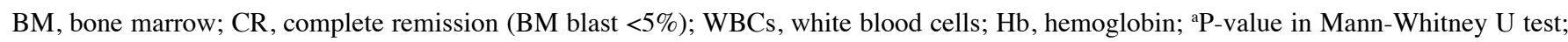
balues shown as median (minimum-maximum).

A

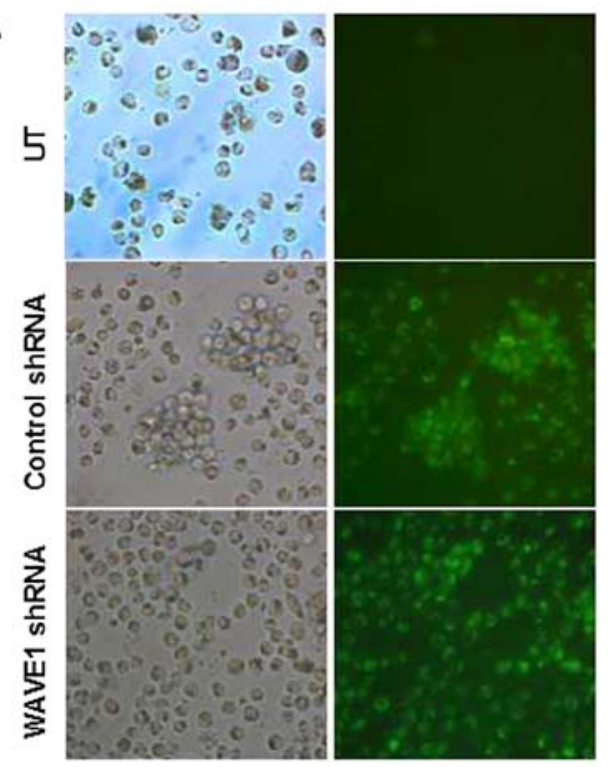

C

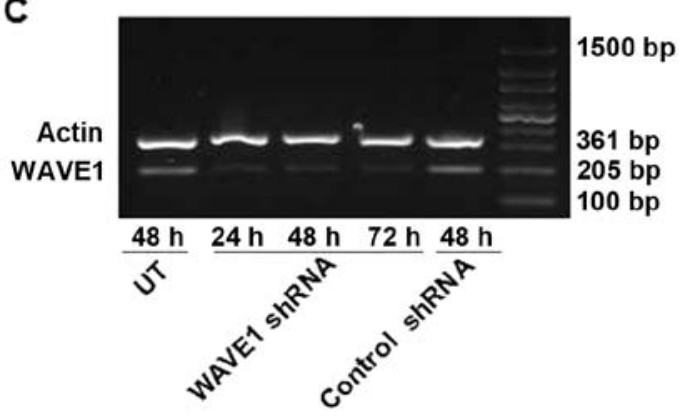

B
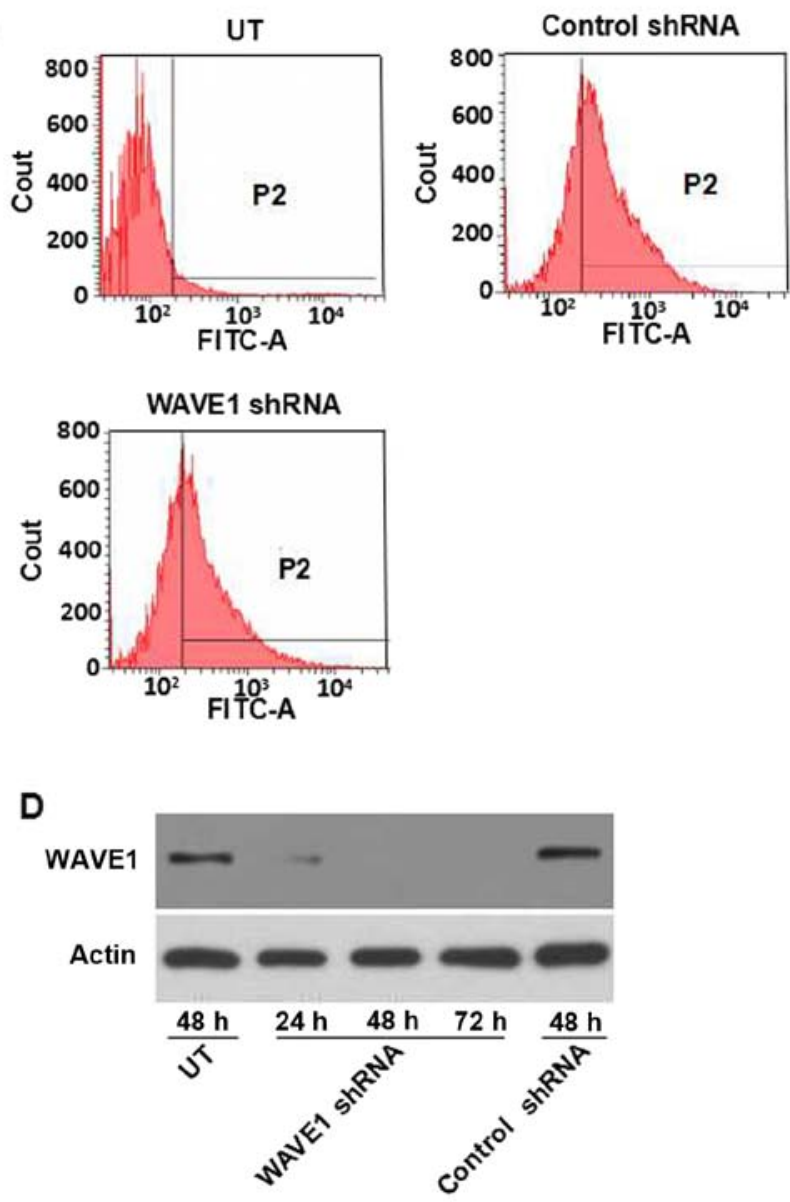

Figure 2. Targeting shRNA-mediated silencing of WAVE1 expression. (A) HL-60 cells were incubated with WAVE1 shRNA and control shRNA transfection for $48 \mathrm{~h}$, and visualized using a fluorescence microscope (magnification, $\mathrm{x} 200$ ). Untransfected cells did not express the vector. (B) Both WAVE1 shRNA and control shRNA were transfected into HL-60 cells. Then transfection efficiency was detected by flow cytometry. (C and D) After transfecting with WAVE1 shRNA or control shRNA for 24, 48 and 72 h, and then WAVE1 levels were, respectively, detected by RT-PCR and western blot analyis in HL-60 cells. Actin was used as a loading control.

blot analysis were performed for detecting the expression of WAVE1. Transfection of WAVE1 shRNA resulted in a marked downregulation of WAVE1. In contrast, transfection of blank vector did not interfere with WAVE1 expression at the level of either mRNA or protein (Fig. 2C and D).

Knockdown of WAVE1 expression inhibits the initiation of autophagy. As a dynamic process for degrading such cytosolic components as dysfunctional organelles and proteins, autophagy offered a pathway of generating metabolic substrates $(28,29)$. A host of existing cancer therapeutics, including DNA-damaging chemotherapy, radiation therapy and molecular targeted therapies, could induce autophagy in cell culture and animal models $(6,30)$. To investigate whether WAVE1 is a direct activator of autophagy, immunoblot was employed for determining microtubule-associated protein light chain 3 (LC3) and its conversion products (LC3-I to LC3-II) (31). With a depletion of WAVE1 expression, 


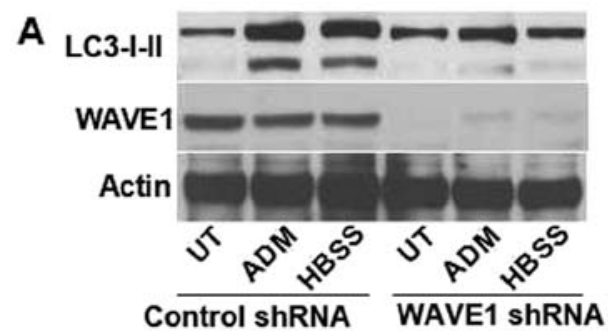

C

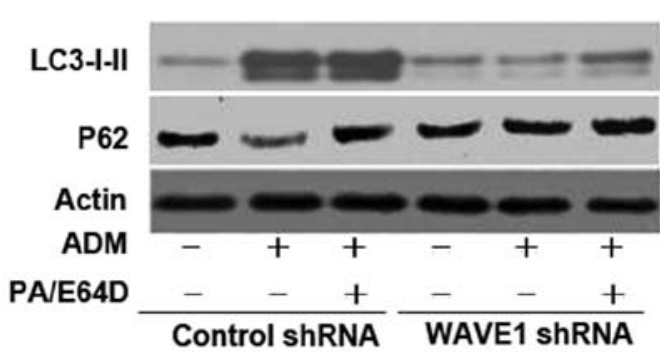

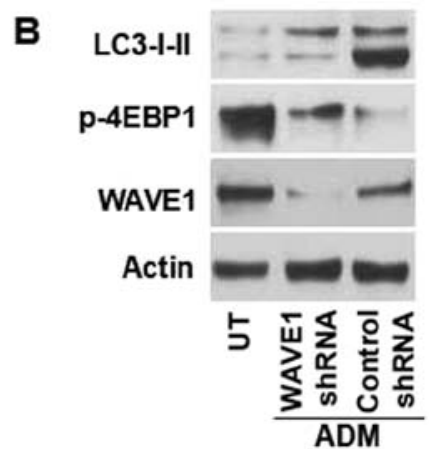

D

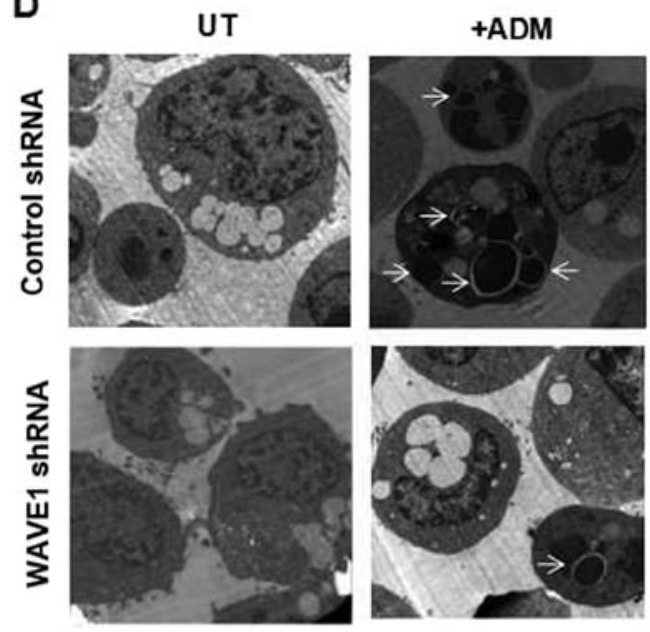

Figure 3. Knockdown of WAVE1 expression inhibits the initiation of autophagy in leukemia cells. (A) After transfection with WAVE1 shRNA or control shRNA for $48 \mathrm{~h}$, HL-60 cells were then treated with ADM $(1 \mu \mathrm{g} / \mathrm{ml})$ and HBSS for $48 \mathrm{~h}$. The levels of LC3-I/II and WAVE1 were assayed by western blot analysis; (B) HL-60 cells were pre-treated with WAVE1 shRNA transfection and then treated with ADM (1 $\mu \mathrm{g} / \mathrm{ml})$ for $48 \mathrm{~h}$. The levels of LC3-I/II and p-4EBP1 were assayed by western blot analysis. (C) After transfecting with WAVE1 shRNA or control shRNA for 48 h, HL-60 cells were treated with ADM $(1 \mu \mathrm{g} / \mathrm{ml})$ for $72 \mathrm{~h}$ with or without pepstatin A (PA, $10 \mu \mathrm{M})$ and E64D $(10 \mu \mathrm{M})$ pretreatment. The levels of LC3-I/II and p62 were assayed by western blot analysis. (D) Ultrastructural features with WAVE1 shRNA transfection for ADM $(1 \mu \mathrm{g} / \mathrm{ml})$ treatment. The number of autophagosomes seen in cells treated with control shRNA was higher than cells treated with WAVE1 shRNA; (magnification, x20,000). White arrows, autophagosomes; UT, untreated group.

the classical autophagic stimuli, such as starvation (Hank's balanced salt solution, HBSS), decreased the expression of LC3-II versus control groups (Fig. 3A). Similar to HBSS, $\mathrm{ADM}$ at a therapeutic dose of $1 \mu \mathrm{g} / \mathrm{ml}$ also decreased LC3-II expression (Fig. 3A), suggesting targeted deletion of WAVE1 inhibited ADM-induced autophagy.

The inhibition of mammalian target of rapamycin (mTOR) signaling pathway has been considered as an important step in the initiation of autophagy $(6,29,32)$, and mTOR activity was further determined through monitoring 4EBP1 (a mTOR substrate) phosphorylation (33). The levels of phosphor-4EBP1 (p-4EBP1) decreased in transfection vector control while a depletion of WAVE1 expression partially rescued p-4EBP1 expression compared with control group (Fig. 3B). An important method for detecting autophagic flux is measuring enhanced degradation of p62 (sequestosome-1), a long-lived scaffolding protein involved in the transport of ubiquitinated protein for proteasomal digestion (34). p62 has LC3 binding domains targeting this protein for incorporation into autophagosome. Thus, it served as a selective substrate of autophagy. Knockdown of WAVE1 decreased the level of LC3-II, yet, the level of p62 increased compared with control group. It suggested that p62 degradation was dependent on WAVE1induced autophagy (Fig. 3C). Moreover, LC3 accumulation and p62 expression were exaggerated after treatment with lysosomal protease inhibitor E64d and pepstatin A vs. WAVE1 shRNA group (Fig. 3C). Thus, the resulting elevation of LC3-II was not due to a decreased degradation of lipidated LC3, but rather to a higher autophagic flux.

The most reliable and conventional technique for visualizing autophagic vacuolization is transmission electron microscopy capable of revealing the presence of multiple autophagosome-like vacuoles with double-membrane structures (31). Ultrastructural analysis revealed that HL-60 cells exhibited fewer autophagosomes after WAVE1 RNAi treatment vs. control group (Fig. 3D). Altogether, these data demonstrate that WAVE1 is required for initiating autophagy in leukemia cells.

Knockdown of WAVE1 expression increases chemotherapy sensitivity. Chemoresistance has become a major obstacle for successful therapeutics of leukemia. A growing body of evidence has suggested that autophagy is an important resistance mechanism for chemotherapy in hematological malignancies (35-37). To characterize the role of WAVE1mediated autophagy in chemosensitivity of leukemia cells, HL-60 cells were treated with several common chemotherapeutic drugs such as ADM $(1 \mu \mathrm{g} / \mathrm{ml})$, VCR $(1 \mu \mathrm{g} / \mathrm{ml})$ and Ara-C $(0.2 \mu \mathrm{M})(32)$. After transfection with WAVE1 shRNA or control shRNA, autophagy was suppressed by 3-MA, a PI3K 
A

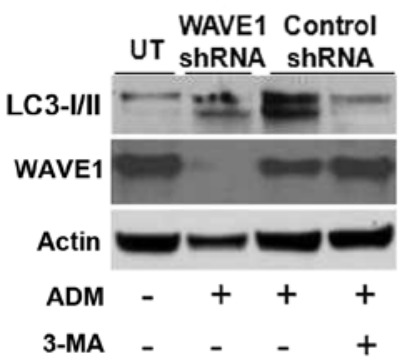

B
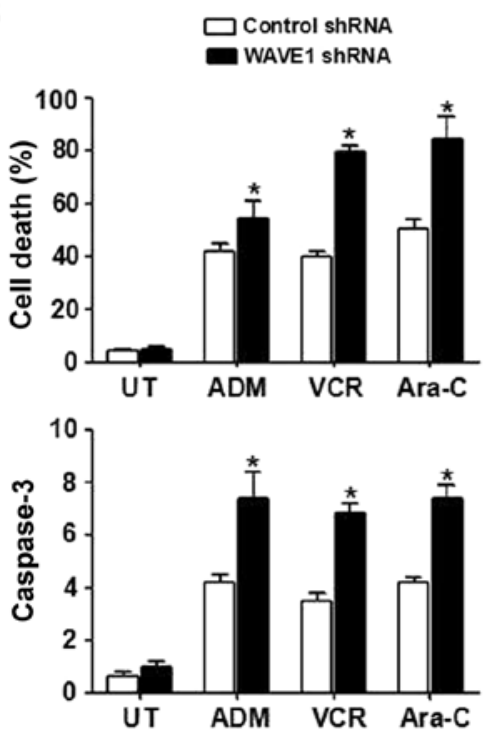

c
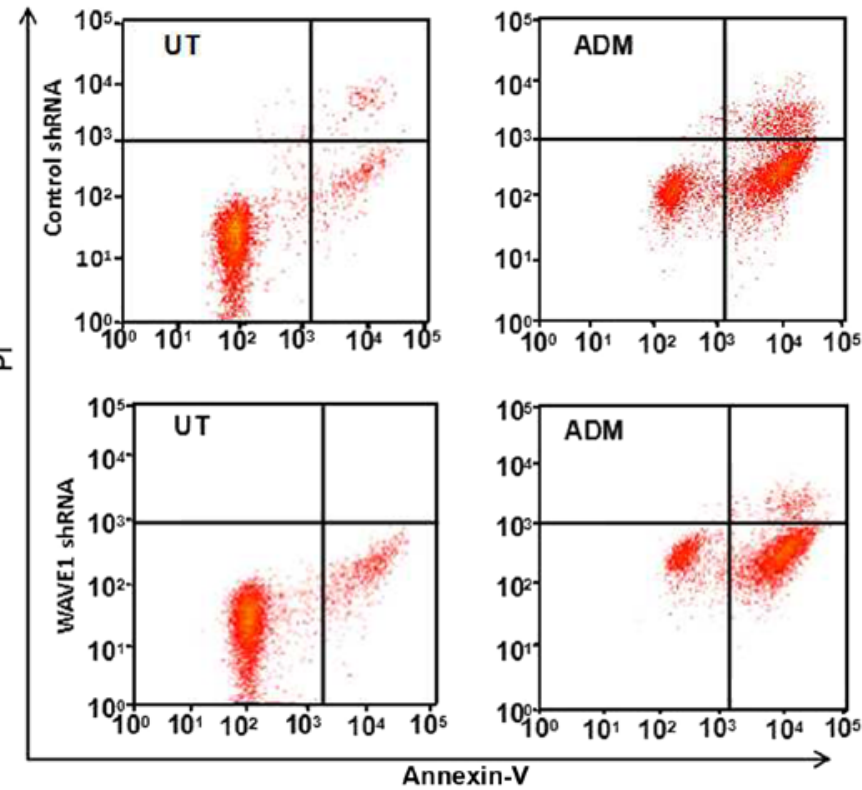

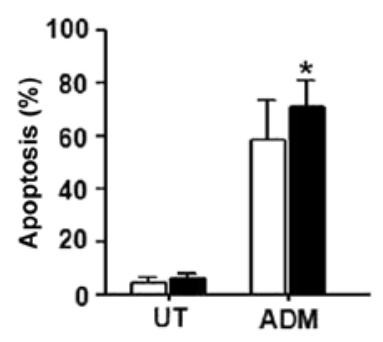

Figure 4. The knockdown of WAVE1 expression increases chemotherapy sensitivity in HL-60 cells. (A) HL-60 cells were pre-treated with WAVE1 shRNA and control shRNA transfection and then treated with ADM $(1 \mu \mathrm{g} / \mathrm{ml})$ for $48 \mathrm{~h}$. The levels of LC3-I/II and WAVE1 were assayed by western blot analysis. LC3 expression by western blot analysis in the presence or absence of 3-MA $(10 \mathrm{mM})$ treatment for $1 \mathrm{~h}$ after control shRNA transfection for $48 \mathrm{~h}$, followed by treatment with ADM for $2 \mathrm{~h}$. (B) WAVE1 knockdown increased the sensitivity of leukemia cells to chemotherapy. After the knockdown of WAVE1 expression by gene transfection and the cells were treated with adriamycin (ADM, $1 \mu \mathrm{g} / \mathrm{ml}$ ), vincristine (VCR, $1 \mu \mathrm{g} / \mathrm{ml}$ ) and cytosine arabinoside (Ara-C, $0.2 \mu \mathrm{M}$ ) for $48 \mathrm{~h}$, cell viability was assayed by MTT assay. ${ }^{*} \mathrm{P}<0.05 \mathrm{vs}$. control shRNA group. (C) WAVE1 knockdown increased anticancer drug-induced apoptosis. After knockdown of WAVE1 expression by gene transfection in HL-60 cells for $48 \mathrm{~h}$ and treatment with ADM (1 $\mu \mathrm{g} / \mathrm{ml})$, early apoptosis was examined by flow cytometry at $48 \mathrm{~h} . \mathrm{P}<0.05$ vs. control shRNA group.

inhibitor. The expression of LC3-II significantly decreased in control group. Similar to using 3-MA, a depletion of WAVE1 expression also decreased LC3-II expression, suggesting that WAVE1 was required for ADM-induced autophagy (Fig. 4A). Moreover, a knockdown of WAVE1 expression in HL-60 cells rendered it significantly more sensitive to ADM, VCR and Ara-C-induced apoptosis associated with high levels of caspase-3 activities (Fig. 4B). Furthermore, a depletion of WAVE1 expression increased early apoptosis with ADM treatment vs. control group (Fig. 4C), supporting a potential prosurvival role for WAVE1-induced autophagy in leukemia cells under chemotherapy.

WAVE1 regulates autophagy in Beclinl/Bcl-2 and Beclin1/ PI3K-III-dependent pathway. WAVE1 is expressed abundantly in mitochondria of neuronal cells (37). Similar to neuronal cells, our previous study showed that WAVE1 was localized to mitochondria and cytoplasm in leukemia cells (10). In the present study the expression of WAVE1 was confirmed by western blot analysis in both mitochondria and cytoplasm (Fig. 5A). Its mitochondrial localization provided a basis for its molecular interaction with different mitochondrial proteins. Bcl-2, an integral membrane protein on endoplasmic reticulum (ER) and mitochondria, is an important inhibitor of apoptosis. Yet, its mechanism of localization is not fully understood (38). WAVE1 knockdown decreased the mitochondrial levels of (Mit)-Bcl-2, but increased the cytoplasmic levels of Bcl-2 (Fig. 5B). Thus, a specific role for WAVE1 was evident in the regulation of Bcl-2 intracellular localization in leukemia cells.

An important molecular event in autophagic vesicle nucleation is the disassociation of Bcl-2-Beclin1 complex and the formation of Beclin1-PI3K-III complex $(31,40)$. The disassociation of Bcl-2-Beclin1 complex sustained autophagy (39). In the present study knockdown of WAVE1 blocked the disassociation of Beclin1-Bcl-2 during enhanced autophagy (Fig. 5D). As PI3K-III is required for autophagy initiation (40), the role of PI3K-III was examined in the regulation of WAVE1-mediated autophagy using a targetspecific shRNA against PI3K-III expression. Transfection of PI3K-III-shRNA led to a significant decrease in PI3K-III protein and significantly inhibited WAVE1-mediated autophagy (Fig. 5C), suggesting that PI3K-III is required for WAVE1-mediated autophagy. Depletion of WAVE1 expres- 

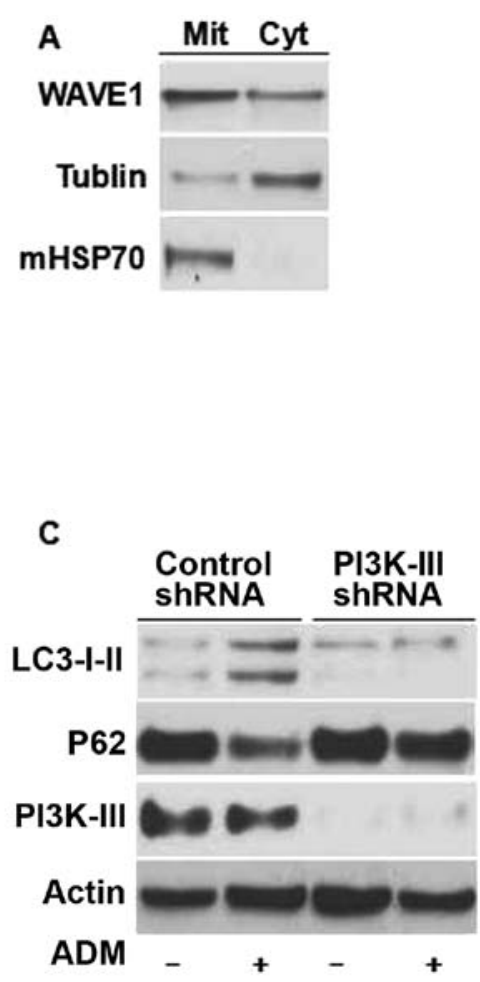

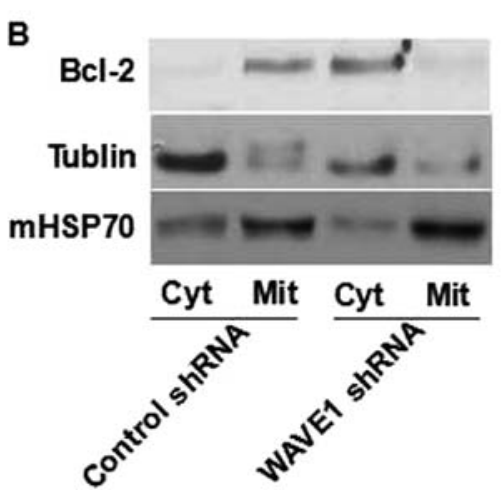

D

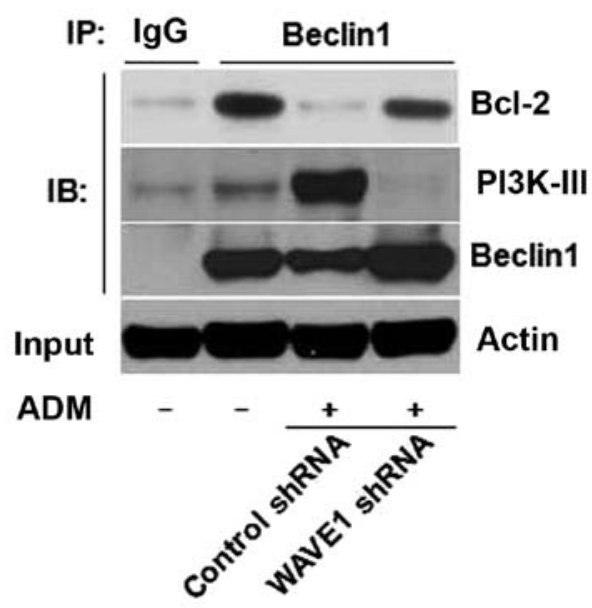

Figure 5. WAVE1 regulates autophagy in Beclin1/Bcl-2 and Beclin1/PI3K-III-dependent pathway. (A) Western blot analysis of WAVE1 in cytoplasmic and mitochondrial fractions of HL-60 cells. The successful separation of cytoplasmic (Cyt) and mitochondria (Mit) fraction was confirmed by western blot analysis of each fraction for known cytoplasmic (tubulin), and mitochondria (mHSP70). (B) Western blot analysis of Bcl-2 level in cytoplasmic (Cyt) and mitochondria (Mit) fractions of HL-60 cells with or without WAVE shRNA transfection. Tubulin and mHSP70 were used as fraction isolation quality control. (C) After transfecting with PI3K-III shRNA or control shRNA for $48 \mathrm{~h}, \mathrm{HL}-60$ cells were treated with ADM $(1 \mu \mathrm{g} / \mathrm{ml})$ for $48 \mathrm{~h}$. The levels of PI3K-III, LC3-I/II and p62 were assayed by western blot analysis. (D) After transfecting with WAVE1 shRNA or control shRNA for $48 \mathrm{~h}, \mathrm{HL}-60$ cells were treated with ADM ( $1 \mu \mathrm{g} / \mathrm{ml})$ for $48 \mathrm{~h}$. Then cell lysates were prepared for immunoprecipitation ('IP') as indicated. The resulting immune complexes and inputs were analyzed by western blot analysis ('IB') as indicated.

sion blocked the interaction between Beclin1 and PI3K-III (Fig. 5D), suggesting that WAVE1 promotes vesicle nucleation. The overall data indicate that WAVE1-mediated autophagy may occur through Beclin1/Bcl-2 and Beclin1/PI3K-IIIdependent pathway in HL-60 cells.

\section{Discussion}

A novel function of WAVE1 is to modulate chemotherapy sensitivity by inducing autophagy in leukemia cells. WAVE1 expression was positively correlated with clinical status in pediatric AML. WAVE1 expression was significantly higher in active phase (such as in primary and relapsing phase) and returned to normal in CR. It was also expressed abundantly in various leukemia cell lines, yet scantly in non-hematological cell lines. Thus, a potentially contributory role of WAVE1 was implicated for the pathogenesis of AML.

Currently chemoresistance is a major obstacle to successful therapeutics of leukemia. Multiple mechanisms of drug resistance are well-recognized, such as drug export transporters (e.g. permeability-glycoprotein), more effective DNA repair, altered pharmacokinetics, resistant hematopoietic stem cells and resistance to apoptosis $(30,32,41)$. Although these mechanisms of drug resistance have been proposed, exact mechanisms remain to be established. Detailed mechanistic understandings of leukemia might help to predict and overcome drug resistance through enhancing chemotherapy and ultimately improving the patient outcomes. WAVE1 has been found to be involved in multidrug resistance and oxidative stress of human leukemia cells, however, its pathogenic role in leukemia was poorly elucidated $(26,42)$. WAVE1 enhanced leukemia cell chemoresistance potentially through the regulation of P-glycoprotein expression (25). Moreover, WAVE1 was associated with mitochondrial $\mathrm{Bcl}-2$, and its depletion caused a mitochondrial release of $\mathrm{Bcl}-2$ and phosphorylation of ASK1/JNK and Bcl-2 in leukemia cells (10). In the present study, it was demonstrated that a knockdown of WAVE1 expression could sensitize leukemia cells to chemotherapeutic drugs and apoptosis. While WAVE1 is essential for leukemic apoptosis, its role in the regulation of leukemia autophagy for drug resistance has been poorly defined. In general, autophagy is a 'programmed cell survival' mechanism because cells employ autophagy for preventing an accumulation of damaged or unnecessary components, but also functions to facilitate the recycling of these components for sustaining homeostasis. Autophagy and apoptosis are currently regarded as different 
aspects of the same cell death continuum, their regulations are intimately connected, and the same regulators could sometimes control both apoptosis and autophagy (43). The role of WAVE1 in cell apoptosis is expected to shed some light also on its role in autophagy.

Numerous studies have demonstrated that autophagy combats various types of adverse stresses and maintains the survival of tumor cells. As a programmed cell survival mechanism responding to cytotoxic chemotherapy or irradiation, it is responsible for drug resistance (7). For example, a loss of autophagy-related genes resulted in the sensitization of resistant carcinoma cells to radiation (44). Furthermore, combined application of chloroquine (an autophagy inhibitor) and imatinib significantly increased the death rate of chronic myeloid leukemia cells, even in imatinib-resistant cases (35). Even though autophagy has been functionally connected with the chemoresistance of tumor cells, little is known about its impact on chemoresistance and its connection with WAVE1 in leukemia cells. The present study demonstrated that WAVE1 is a direct regulator of autophagy in leukemia cells thereby controlling LC3 conversion (LC3-I to LC3-II), a degradation of p62 and a maturation of autophagosomes. Moreover, a depletion of WAVE1 expression inhibited autophagy, promoted sensitivity of leukemia cells to chemotherapeutic drugs and enhanced apoptosis. There was a dominant role of WAVE1 in regulating autophagy, and chemoresistance of leukemia cells has been highly correlated with autophagy.

The process of mammalian autophagy is divided into several steps of initiation, nucleation, elongation, closure, maturation and degradation or extrusion (45). As a key regulator of autophagy, Beclin1 is a core component of class III $\mathrm{PI} 3 \mathrm{~K} / \mathrm{Vps} 34$ complex required for autophagosomal formation and maturation (46), and it interacts with autophagy regulators, organelle membrane anchor proteins, Bcl-2 and Bcl-xL. The disassociation of Bcl-2-Beclin1 complex plays an important role in autophagic vesicle nucleation $(39,46)$. As an integral membrane protein on ER and within mitochondria, Bcl-2 is an important inhibitor of apoptosis. WAVE1 might function as an anchoring protein for mitochondrial Bcl-2 so as to affect the mitochondrial release and phosphorylation of Bcl-2 (10). WAVE1 was localized to mitochondria of leukemia cells and a knockdown of WAVE1 expression induced the translocation of Bcl-2 from mitochondria into the cytoplasm. Moreover, WAVE1 potentially confers its pro-autophagic activities by controlling Beclin1-Bcl-2 complex formation through disrupting the interactions between Beclin1 and Bcl-2. Thus, the disassociation of Beclin1-Bcl-2 complex is an important mechanism of WAVE1-regulated autophagy.

PI3K (PI3-kinase/PI3K) family is divided into three classes of I, II and III. PI3K-III activity was essential for autophagy whereas PI3K-I activity had some inhibitory effects on autophagy (31). PI3K-III, a mammalian homolog of Vps34, was first identified in yeast. It has been implicated in such a wide range of cellular phenomena as autophagy, phagocytosis and post-endocytic receptor sorting $(40,47,48)$. In yeast models, Vps34/class III PI3K formed macromolecular complexes with autophagic protein Apg6/Beclin1 (46). It was found that genetic inhibition of PI3K-III by specific
shRNA suppressed ADM-induced autophagy, suggesting that PI3K-III activity is required for ADM-induced autophagy. Furthermore, a depletion of WAVE1 expression suppressed the interaction between Beclin1 and PI3K-III. There was a dominating role of WAVE1 in regulating Beclin1-PI3K-III complex formation of autophagy.

In summary, overexpression of WAVE1 is an unfavorable prognostic factor in pediatric AML and leukemia cells. As a positive regulator of autophagy, it can enhance the chemoresistance of leukemia cells and regulate the formative autophagosomal interaction of Beclin1-Bcl-2 and Beclin1PI3K-III complexes. The above findings further confirm its roles in autophagy and chemoresistance of leukemia cells.

\section{Acknowledgements}

The present study was supported by grants from the National Natural Science Foundation of China (grant nos. 81400138, 31171328 and 81270616) and the Guang Dong Provincial Medical Scientific Research Foundation (grant no. A2013585).

\section{References}

1. Abrahamsson J, Forestier E, Heldrup J, Jahnukainen K, Jónsson OG, Lausen B, Palle J, Zeller B and Hasle H: Responseguided induction therapy in pediatric acute myeloid leukemia with excellent remission rate. J Clin Oncol 29: 310-315, 2011.

2. Felice MS, Rossi JG, Alonso CN, Gallego MS, Eberle SE, Alfaro EM, Guitter MR, Bernasconi AR, Rubio PL, Coccé MC, et al: Experience with four consecutive BFM-based protocols for treatment of childhood with non-promyelocytic acute yeloblastic leukemia in Argentina. Leuk Lymphoma 6: 1-10, 2016 (Epub ahead of print).

3. Gibson BE, Wheatley K, Hann IM, Stevens RF, Webb D, Hills RK, De Graaf SS and Harrison CJ: Treatment strategy and long-term results in paediatric patients treated in consecutive UK AML trials. Leukemia 19: 2130-2138, 2005.

4. Sander A, Zimmermann M, Dworzak M, Fleischhack G, von Neuhoff C, Reinhardt D, Kaspers GJ and Creutzig U: Consequent and intensified relapse therapy improved survival in pediatric AML: Results of relapse treatment in 379 patients of three consecutive AML-BFM trials. Leukemia 24: 1422-1428, 2010.

5. Kaspers GJL, Zimmermann M, Reinhardt D, Gibson BES, Tamminga RYJ, Aleinikova O, Armendariz H, Dworzak M, Ha SY, Hasle H, et al: Improved outcome in pediatric relapsed acute myeloid leukemia: Results of a randomized trial on liposomal daunorubicin by the International BFM Study Group. J Clin Oncol 31: 599-607, 2013.

6. White E and DiPaola RS: The double-edged sword of autophagy modulation in cancer. Clin Cancer Res 15: 5308-5316, 2009.

7. Shimizu S: Development of anti-cancer drugs mediated by apoptosis and autophagy. Nihon Rinsho 73: 1302-1307, 2015 (In Japanese).

8. Takenawa T and Suetsugu S: The WASP-WAVE protein network: Connecting the membrane to the cytoskeleton. Nat Rev Mol Cell Biol 8: 37-48, 2007.

9. Gourlay CW, Carpp LN, Timpson P, Winder SJ and Ayscough KR: A role for the actin cytoskeleton in cell death and aging in yeast. J Cell Biol 164: 803-809, 2004.

10. Kang $\mathrm{R}$, Tang $\mathrm{D}, \mathrm{Yu} \mathrm{Y}$, Wang $\mathrm{Z}, \mathrm{Hu} \mathrm{T}$, Wang $\mathrm{H}$ and Cao L: WAVE1 regulates Bcl-2 localization and phosphorylation in leukemia cells. Leukemia 24: 177-186, 2010.

11. Monastyrska I, Rieter E, Klionsky DJ and Reggiori F: Multiple roles of the cytoskeleton in autophagy. Biol Rev Camb Philos Soc 84: 431-448, 2009

12. Di Bartolomeo S, Corazzari M, Nazio F, Oliverio S, Lisi G, Antonioli M, Pagliarini V, Matteoni S, Fuoco C, Giunta L, et al: The dynamic interaction of AMBRA1 with the dynein motor complex regulates mammalian autophagy. J Cell Biol 191: 155-168, 2010. 
13. Batlevi Y, Martin DN, Pandey UB, Simon CR, Powers CM, Taylor JP and Baehrecke EH: Dynein light chain 1 is required for autophagy, protein clearance, and cell death in Drosophila. Proc Natl Acad Sci USA 107: 742-747, 2010.

14. Wrighton KH: Autophagy: Myosin II moves in on autophagosomes. Nat Rev Mol Cell Biol 12: 77, 2011.

15. Cardoso CM, Groth-Pedersen L, Høyer-Hansen M, Kirkegaard T, Corcelle E, Andersen JS, Jäättelä M and Nylandsted J: Depletion of kinesin 5B affects lysosomal distribution and stability and induces peri-nuclear accumulation of autophagosomes in cancer cells. PLoS One 4: e4424, 2009.

16. Tang HW, Wang YB, Wang SL, Wu MH, Lin SY and Chen GC: Atg1-mediated myosin II activation regulates autophagosome formation during starvation-induced autophagy. EMBO J 30: 636-651, 2011.

17. Jahreiss L, Menzies FM and Rubinsztein DC: The itinerary of autophagosomes: From peripheral formation to kiss-and-run fusion with lysosomes. Traffic 9: 574-587, 2008.

18. Fass E, Shvets E, Degani I, Hirschberg K and Elazar Z: Microtubules support production of starvation-induced autophagosomes but not their targeting and fusion with lysosomes. J Biol Chem 281: 36303-36316, 2006.

19. Miki H, Suetsugu S and Takenawa T: WAVE, a novel WASPfamily protein involved in actin reorganization induced by Rac. EMBO J 17: 6932-6941, 1998.

20. Machesky LM and Insall RH: Scarl and the related WiskottAldrich syndrome protein, WASP, regulate the actin cytoskeleton through the Arp2/3 complex. Curr Biol 8: 1347-1356, 1998.

21. Suetsugu S, Miki H and Takenawa T: Identification of two human WAVE/SCAR homologues as general actin regulatory molecules which associate with the Arp2/3 complex. Biochem Biophys Res Commun 260: 296-302, 1999.

22. Dahl JP, Wang-Dunlop J, Gonzales C, Goad ME, Mark RJ and Kwak SP: Characterization of the WAVE1 knock-out mouse: Implications for CNS development. J Neurosci 23: 3343-3352, 2003.

23. Rawe VY, Payne C, Navara C and Schatten G: WAVE1 intranuclear trafficking is essential for genomic and cytoskeletal dynamics during fertilization: Cell-cycle-dependent shuttling between M-phase and interphase nuclei. Dev Biol 276: 253-267, 2004.

24. Yamaguchi $\mathrm{H}$ and Condeelis $\mathrm{J}$ : Regulation of the actin cytoskeleton in cancer cell migration and invasion. Biochim Biophys Acta 1773: 642-652, 2007.

25. Yang MH, Zhao MY, Wang Z, Kang R, He YL, Yin XC, Liu LY, Yang LC, Zhan CX, Wu XS, et al: WAVE1 regulates P-glycoprotein expression via Ezrin in leukemia cells. Leuk Lymphoma 52: 298-309, 2011.

26. Kang R, Cao LZ, Yu Y, Hu T, Wang Z, Xu WQ and Xie M: Role of WAVE1 in drug resistance of K562/A02 leukemia cells. Zhonghua Xue Ye Xue Za Zhi 28: 379-382, 2007 (In Chinese).

27. Thorburn A: Apoptosis and Autophagy: regulatory connections between two supposedly different processes. Apoptosis 13: 1-9, 2008.

28. Klionsky DJ and Emr SD: Autophagy as a regulated pathway of cellular degradation. Science 290: 1717-1721, 2000.

29. Levine B. Cell biology: Autophagy and cancer. Nature 446: 745-747, 2007

30. Liu L, Yang M, Kang R, Wang Z, Zhao Y, Yu Y, Xie M, Yin X, Livesey KM, Loze MT, et al: DAMP-mediated autophagy contributes to drug resistance. Autophagy 7: 112-114, 2011.

31. Mizushima N, Yoshimori T and Levine B: Methods in mammalian autophagy research. Cell 140: 313-326, 2010.
32. Yang L, Yu Y, Kang R, Yang M, Xie M, Wang Z, Tang D, Zhao M, Liu L, Zhang H, et al: Up-regulated autophagy by endogenous high mobility group box-1 promotes chemoresistance in leukemia cells. Leuk Lymphoma 53: 315-322, 2012.

33. Klionsky DJ, Abeliovich H, Agostinis P, Agrawal DK, Aliev G, Askew DS, Baba M, Baehrecke EH, Bahr BA, Ballabio A, et al: Guidelines for the use and interpretation of assays for monitoring autophagy in higher eukaryotes. Autophagy 4: 151-175, 2008.

34. Pankiv S, Clausen TH, Lamark T, Brech A, Bruun JA, Outzen H, Øvervatn A, Bjørkøy G and Johansen T: p62/SQSTM1 binds directly to Atg8/LC3 to facilitate degradation of ubiquitinated protein aggregates by autophagy. J Biol Chem 282: 24131-24145, 2007.

35. Bellodi C, Lidonnici MR, Hamilton A, Helgason GV, Soliera AR, Ronchetti M, Galavotti S, Young KW, Selmi T, Yacobi R, et al: Targeting autophagy potentiates tyrosine kinase inhibitorinduced cell death in Philadelphia chromosome-positive cells, including primary CML stem cells. J Clin Invest 119: 1109-1123, 2009.

36. Sehgal AR, Konig H, Johnson DE, Tang D, Amaravadi RK, Boyiadzis M and Lotze MT: You eat what you are: Autophagy inhibition as a therapeutic strategy in leukemia. Leukemia 29: 517-525, 2015.

37. Sung JY, Engmann O, Teylan MA, Nairn AC, Greengard P and Kim Y: WAVE1 controls neuronal activity-induced mitochondrial distribution in dendritic spines. Proc Natl Acad Sci USA 105: 3112-3116, 2008.

38. Hockenbery D, Nuñez G, Milliman C, Schreiber RD and Korsmeyer SJ: Bcl-2 is an inner mitochondrial membrane protein that blocks programmed cell death. Nature 348: 334-336, 1990.

39. Pattingre S, Tassa A, Qu X, Garuti R, Liang XH, Mizushima N, Packer M, Schneider MD and Levine B: Bcl-2 antiapoptotic proteins inhibit Beclin 1-dependent autophagy. Cell 122: 927-939, 2005.

40. Petiot A, Ogier-Denis E, Blommaart EFC, Meijer AJ and Codogno P: Distinct classes of phosphatidylinositol 3'-kinases are involved in signaling pathways that control macroautophagy in HT-29 cells. J Biol Chem 275: 992-998, 2000.

41. Sakamoto KM, Grant S, Saleiro D, Crispino JD, Hijiya N, Giles F, Platanias L and Eklund EA: Targeting novel signaling pathways for resistant acute myeloid leukemia. Mol Genet Metab 114: 397-402, 2015.

42. He YL, Cao LZ, Yang J, Yang MH, Xu WQ, Xie M and Shi Z: Expression of WAVE1 and p22phox in children with acute lymphocytic leukemia and the relationship of WAVE1 with oxidative stress. Zhongguo Dang Dai Er Ke Za Zhi 11: 88-92, 2009 (In Chinese).

43. Abraham MC and Shaham S: Death without caspases, caspases without death. Trends Cell Biol 14: 184-193, 2004.

44. Apel A, Herr I, Schwarz H, Rodemann HP and Mayer A: Blocked autophagy sensitizes resistant carcinoma cells to radiation therapy. Cancer Res 68: 1485-1494, 2008.

45. Yang Z and Klionsky DJ: Eaten alive: A history of macroautophagy. Nat Cell Biol 12: 814-822, 2010.

46. Sinha S and Levine B: The autophagy effector Beclin 1: A novel BH3-only protein. Oncogene 27 (Suppl 1): S137-S148, 2008.

47. Fratti RA, Backer JM, Gruenberg J, Corvera S and Deretic V: Role of phosphatidylinositol 3-kinase and Rab5 effectors in phagosomal biogenesis and mycobacterial phagosome maturation arrest. J Cell Biol 154: 631-644, 2001.

48. Siddhanta U, McIlroy J, Shah A, Zhang Y and Backer JM: Distinct roles for the p110alpha and hVPS34 phosphatidylinositol 3'-kinases in vesicular trafficking, regulation of the actin cytoskeleton, and mitogenesis. J Cell Biol 143: 1647-1659, 1998. 